\title{
In vivo monitoring of the potassium channel KcsA in Streptomyces lividans hyphae using immuno- electron microscopy and energy-filtering transmission electron microscopy
}

\author{
Jan Hegermann, Jens Overbeck and Hildgund Schrempf \\ FB Biologie/Chemie, Universität Osnabrück, Barbarastr. 11, D-49069 Osnabrück, Germany
}

Correspondence

Hildgund Schrempf

schrempf@

biologie.uni-osnabrueck.de

Received 16 March 2006

Accepted 14 May 2006
The previous discovery of the Streptomyces lividans kcsA gene and its overexpression followed by the functional reconstitution of the purified gene product has resulted in new strategies to explore this channel protein in vitro. KcsA has evolved as a general model to investigate the structure/ function relationship of ion channel proteins. Using specific antibodies raised against a domain of KcsA lacking membrane-spanning regions, KcsA has now been localized within numerous separated clusters between the outer face of the cytoplasm and the cell envelope in substrate hyphae of the $S$. lividans wild-type strain but not in a designed chromosomal disruption mutant $\Delta \mathrm{K}$, lacking a functional $k c s A$ gene. Previous findings had revealed that caesium ions led to a block of KcsA channel activity within S. lividans protoplasts fused to giant vesicles. As caesium can be scored by electron energy loss spectroscopy better than potassium, this technique was applied to hyphae that had been briefly exposed to caesium instead of potassium ions. Caesium was found preferentially at the cell envelope. Compared to the $\Delta \mathrm{K}$ mutant, the relative level of caesium was $\approx 30 \%$ enhanced in the wild-type. This is attributed to the presence of KcsA channels. Additional visualization by electron spectroscopic imaging supported this conclusion. The data presented are believed to represent the first demonstration of in vivo monitoring of KcsA in its original host.

\section{INTRODUCTION}

The lifecycle of streptomycetes is initiated by the germination of spores. Subsequent extension of the hyphae and branching leads to the formation of an extended filamentous network, the substrate mycelium. Upon depletion of nutrients aerial hyphae arise; within these hyphae, septa are formed, and then the resulting compartments containing genomic DNA develop into spores. These withstand dryness as well as cold conditions and rest in a dormant stage as long as nutrients are not available. Within the natural habitat streptomycetes play a major role in the turnover of organic material as they have a large repertoire of enzymes leading to the degradation of macromolecules including cellulose, lignocellulose, chitin, starch, xylan, proteins and lipids. Hence these enzymes are of great ecological and biotechnological value. Streptomycetes have a huge capacity to synthesize secondary metabolites, many of which have antibacterial, antifungal or cytostatic activities and are being

Abbreviations: EELS, electron energy loss spectroscopy; EFTEM, energy-filtering transmission electron microscopy; ESI, electron spectroscopic imaging; IEM, immuno-electron microscopy. used in medicine (see reviews by Kutzner, 1981; Schrempf, 1999).

Within Streptomyces lividans the kcsA gene, which encodes a protein of $16 \mathrm{kDa}$ with two predicted transmembrane helices, has been identified. Multiple aligments had suggested that KcsA has closest kinship to the P-region and its neighbouring transmembrane helices (S5 and S6) of eukaroytic voltage-gated $\mathrm{K}^{+}$channel families. Investigations of the in vitro reconstituted protein and of a newly established protoplast-vesicle system led to the discovery that KcsA acts as a potassium channel (Schrempf et al., 1995). Being the first discovered bacterial protein functioning as a $\mathrm{K}^{+}$channel and the first one which can be obtained in large quantities (Schrempf et al., 1995), it was subsequently crystallized without its C-terminal domain as a tetramer (Doyle et al., 1998). The discovery of the bacterial KcsA channel has opened new avenues for exploring a channel protein. The increasing number of sequenced genomes led to the deduction of a range of bacterial proteins that could also function as ion channels (Derst \& Karschin, 1998). Using 3D computer modelling, conserved sequences of transmembrane regions as well as the putative pore 
elements within $\mathrm{K}^{+}$transporters and channels have been quantified and classified. Together with the analyses of results from mutational studies it has been concluded that $\mathrm{K}^{+}$symporter families from prokaryotes and eukaryotes have evolved from the prokaryotic $\mathrm{K}^{+}$channel proteins (Durell et al., 1999; Durell \& Guy, 2001). The findings were used to clone and overexpress a few additional bacterial proteins (as done for KcsA) and to generate their crystal structure in order to understand the general principle of ion channels (see review by Doyle, 2004). The results of experimental data (Meuser et al., 2001) support the conclusion that the crystal structure of the tetrameric KcsA does not present the 'open state', as initially concluded (Doyle et al., 1998). Hence several implications for ion permeation (including the hydration status of the permeating $\mathrm{K}^{+}$ion) need to be reinvestigated (Schrempf, 2005). Thus it will be an important future task to generate the crystal structure of the full-length protein trapped within the open state.

Energy-filtering transmission electron microscopy (EFTEM) allows the acquisition of electron energy loss spectroscopy (EELS) information or elemental maps by electron spectroscopic imaging (ESI), both being based on the detection of inelastically scattered electrons within the sample at an element-specific energy loss. The spatial resolution is in the range of $1 \mathrm{~nm}$, when evaluating a series of energy-filtered images around an edge (Hofer et al., 1999). EELS analysis has been adopted to investigate small biological samples (Somlyo \& Shuman, 1982), including granules of vacuoles (Bucking et al., 1998), the apical hair membrane within the cochlea of the guinea pig (Heinrich et al., 1998), root cells of Allium (Liu \& Kottke, 2003), polyphosphate granules in bacteria (Chavez et al., 2004), and spores of bacilli and different structures of mycorrhizal fungi (Kottke, 1991; Cruz, 2004).

To obtain information about the in vivo localization of KcsA within its original host, we generated antibodies and a mutant of S. lividans carrying a disruption of its chromosomal $k c s A$ gene. Using immuno-electron microscopy (IEM) we show that the KcsA channel is arranged in clusters within the cytoplasmic membrane of hyphae of the wildtype S. lividans and that it is absent in the mutant lacking a functional $k c s A$ gene. In addition, EFTEM is shown, apparently for the first time, to be applicable to monitoring differences in ion accumulation in bacteria correlated with the presence or absence of ion channels.

\section{METHODS}

Strains and plasmids. Streptomyces lividans 66 (in the text designated S. lividans) (Hopwood et al., 1985) and Escherichia coli M15 pREP4 (Qiagen), and the E. coli vector pQE32 (Qiagen) and the bifunctional vector pGM160 (Muth et al., 1995) were used.

Media and cultivation of bacteria. E. coli colonies were grown on LB agar or in LB liquid medium (Sambrook et al., 1989). Spores of $S$. lividans were inoculated in complete or minimal media (Schlochtermeier et al., 1992) in baffled Erlenmeyer flasks and cultivated for 24-36 h. To generate spores, S. lividans was grown on complete medium containing agarose until sporulation occurred (Hopwood et al., 1985).

Purchase of chemicals and enzymes. Chemicals for SDS gel electrophoresis were obtained from Serva. Other chemicals were supplied by Sigma.

Cloning of a truncated $k \operatorname{cs} A$ gene, isolation of the fusion protein and generation of antibodies. The vector pQE32 containing the C-terminal part of $k c s A$ gene was described earlier (Schrempf et al., 1995). To generate a truncated gene (encoding only the C-terminal portion) a $217 \mathrm{bp}$ fragment was amplified using Vent polymerase, the primer 1 containing a SphI cleavage site and the primer 2 with a designed HindIII site. The primers had the following sequence: p1, CCGCGCGCATGCCCTGGTTCGTCGGCCG; p2, CGCACAAGCTTCGCAGACTCATCGGTGC.

The resulting fragment was cleaved with SphI and HindIII and ligated into the correspondingly cleaved $\mathrm{pQE} 32$ vector and transformed into E. coli M15 pREP4. The E. coli M15 pREP4 containing the correct construct was inoculated in LB medium with ampicillin $\left(100 \mu \mathrm{g} \mathrm{ml}^{-1}\right)$ and kanamycin $\left(25 \mu \mathrm{g} \mathrm{ml}^{-1}\right)$ and during its exponential growth phase $\left(\mathrm{OD}_{600} 0 \cdot 6\right)$ induced with $1 \mathrm{mM}$ IPTG for $4 \mathrm{~h}$ at $37^{\circ} \mathrm{C}$. Cells were disrupted by sonication and cell debris was removed by centrifugation $(14000 \mathrm{~g})$. Proteins were purified using $\mathrm{Ni}^{2+}$ NTA affinity chromatography. To remove impurities, the procedure was repeated. The isolated protein $(100 \mu \mathrm{g})$ was used to generate antibodies in guinea pigs (EUROGENTEC Belgium). The antisera (from rabbit) were stored in aliquots at $-20^{\circ} \mathrm{C}$.

Preparation and analysis of membrane and cytosolic fractions of E. coli. E. coli M15 pREP4 containing the pQE32 construct with the KcsA or the truncated gene (see above) was grown and induced as described above. However, induction was done for $2 \mathrm{~h}$ only. After disruption of the cells, the debris was removed (see above). The supernatant was subjected to high-speed centrifugation $(10 \mathrm{~min}, 100000 \mathrm{~g}$ ). The pellet (membrane fraction) was washed and suspended in buffer containing dodecylmaltoside (Splitt et al., 2000). The supernatant represents the cytoplasmic fraction. Corresponding aliquots were subjected to SDS-PAGE. A subsequent Western-blot analysis was pursued with the obtained antibodies (see above chapter).

Generation of the $\mathbf{S}$. lividans mutant $\Delta \mathbf{K}$. The previously described vector pGM160 (Muth et al., 1995) is a bifunctional E. coli/Streptomyces vector. Within streptomycetes its replication is temperature-sensitive. It contains an ampicillin-resistance gene (selection in E. coli) and (for selection within streptomycetes) the thiostrepton- and gentamicin-restistance gene (aacC1). The aacC1 gene was replaced (after cleavage by $\mathrm{Ncol}$ and HindIII) by a correspondingly cleaved fragment which was constructed in the following fashion: the Omega-hyg fragment (Blondelet-Rouault et al., 1997) with the hygromycin (hyg)-resistance gene (Fig. 1) was flanked with one PCR-generated $725 \mathrm{bp}$ fragment (comprising $27 \mathrm{bp}$ of $k c s A$ and $698 \mathrm{bp}$ of its upstream region) and another $696 \mathrm{bp}$ fragment (comprising $57 \mathrm{bp}$ of $k c s A$ and $639 \mathrm{bp}$ of its flanking downstream region) according to the previously described procedure (Xiao et al., 2002). The resulting pGM160-based construct was transformed into S. lividans protoplasts (Hopwood et al., 1985). Thiostrepton-resistant colonies were replica-plated $\left(30^{\circ} \mathrm{C}\right)$ onto hygromycin-containing plates. Subsequently, the colonies were replica-plated (twice) on hygromycin-containing plates and incubated at $37^{\circ} \mathrm{C}$. Under these conditions the replication of the designed construct ceases. Hence hygromycin-resistant surviving colonies will usually be the result of double crossing over among regions (flanking the Omega-hyg fragment) and those being homologous with the S. lividans chromsosome (Fig. 1). 


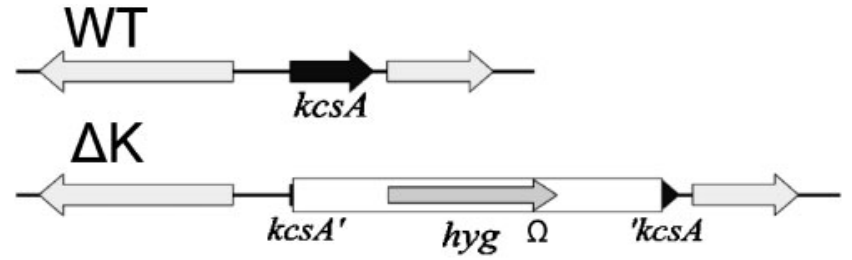

Fig. 1. Relative positioning of the $k c s A$ gene (black) and the neighbouring genes (light grey) within the chromosomal DNA of S. lividans WT, and the Omega-hyg fragment (white box) with the hygromycin-resistance gene (hyg, in grey) followed by a transcriptional terminator $(\Omega)$ flanked by the small residual portions (black) of the $k c s A$ gene (named $k \operatorname{cs} A^{\prime}$ and ' $k c s A$ ) within the DNA of the mutant $\Delta \mathrm{K}$.

EELS of $\mathbf{C s C l}$ crystals. Crystals were obtained on a 400-mesh Formvar-nickel grid by floating it on a drop of saturated aqueous solution of $\mathrm{CsCl}$ for a few seconds and air drying after removal of excess fluid with filter paper. The peak height was monitored as a function of the amount of crystal material included in the measuring point.

Preparation of mycelia for EELS/ESI measurements. Formvar-coated nickel grids were dipped onto a drop of a $0 \cdot 1 \%$ solution of polylysine and blotted dry on filter paper at room temperature. Mycelia were disaggregated by repeated sucking in a Pasteur pipette, and a $30 \mu \mathrm{l}$ drop was pipetted onto a polylysinecoated grid which was then incubated for $10 \mathrm{~min}$ at room temperature in a moisture chamber, allowing settling of the mycelia and their adherence to the grid. Residual medium and excess mycelia were removed from the grid with filter paper. All grids were incubated $30 \mathrm{~min}$ at room temperature, floating on minimal medium containing $100 \mathrm{mM} \mathrm{CsCl}$. Afterwards they were dipped on a $500 \mu \mathrm{l}$ drop of minimal medium containing only $10 \mathrm{mM} \mathrm{CsCl}$ for $10 \mathrm{~s}$ to avoid precipitation of caesium at and around hyphae. Adhering medium was removed carefully with filter paper and the grids were air-dried.

EELS analysis. The EELS analysis was done on a Zeiss-EM 902A equipped with an in-column Henri-Castain electron energy loss spectrometer. Spectra were drawn with an analogue $x-y$ recorder. The cathode was saturated at $80 \mathrm{kV}$ with the cathode-current setting at $1 \mu \mathrm{A}$. The object was positioned in the centre of the fluorescent screen at a magnification of 20000 -fold or 85000 -fold and the condenser was maximally focused. A $100 \mu \mathrm{m}$ spectrometer-entrance aperture was positioned in the centre of the optical path, and an EELS registration area of 0.16 or $0.7 \mu \mathrm{m}$ in diameter was set (depending on the magnification). After correct positioning of the object, electron beam deflection and condenser focus settings were controlled again in order to achieve maximal brightness. The $0 \mathrm{eV}$ point was positioned exactly on the sensor of the photomultiplier tube (PMT). The slit aperture was inserted, adjusted to an energy window of $\sim 15 \mathrm{eV}$, and positioned to the PMT sensor. Spectra obtained by the PMT were recorded between 720 and $770 \mathrm{eV}$ energy loss. The $x-y$ recorder was set to $50 \mathrm{mV}$ in the $x$ - and $10 \mathrm{mV}$ in the $y$-dimension. Caesium is recognized from the spectrogram by two peaks at $726 \mathrm{eV}$ and $740 \mathrm{eV}$ energy loss $\left(\mathrm{M}_{45}\right.$ edges) (Ahn \& Krivanek, 1983). The height of the first peak $(726 \mathrm{eV})$ was measured in $\mathrm{mm}$ as depicted in Fig. 4(a).

Performance of ESI analysis. Specimens were prepared as described for EELS. Analysis was done using EFTEM software (Soft Imaging Systems). Images of $710 \mathrm{eV}$ to $830 \mathrm{eV}$ energy loss were recorded with a cooled $1024 \times 1024$ CCD camera (Proscan CCD 512/1024; Proscan Electronic Systems) and analysed according to the three-windows-white-line method.

Quantitative comparison of EELS data obtained for two strains. Four measuring points of $0.16 \mu \mathrm{m}$ were positioned at the inner margins of hyphae as shown in Fig. 5. This procedure was repeated two to four times within one grid window and the mean peak height was calculated. Then the grid window or the grid was changed. In the same fashion, 100 spectra, subdivided into 25 groups of four, were recorded for each strain. Mean peak height and the standard deviation were calculated.

IEM. After fixation in $0 \cdot 25 \%$ glutaraldehyde and $3 \cdot 7 \%$ formaldehyde, bacteria were embedded in Lowicryl K4M (Roth et al., 1981). Ultrathin sectioning was performed using a Reichert-Jung Ultracut E with a diamond knife (Reichert-Jung Diatome $45^{\circ}$ ). Ultrathin sections of E. coli cells or S. lividans hyphae were mounted on Formvar-nickel grids (300 mesh). Immuno-labelling was done on ultrathin sections of $E$. coli cells or S. lividans hyphae using the above-described anti-KcsA antibodies (first antibody). All incubations were done on $50 \mu$ drops with the grid on top of the drop, the sections facing downwards. Dilution of the antibodies was done in PBST [PBS containing $0 \cdot 025 \%(\mathrm{v} / \mathrm{v})$ Tween 20] supplemented with $0 \cdot 2 \%(\mathrm{w} / \mathrm{v}) \mathrm{BSA}$. The grids were first incubated for $30 \mathrm{~min}$ on drops of PBS containing $2 \%(\mathrm{w} / \mathrm{v}) \mathrm{BSA}$, then $3 \mathrm{~h}$ on serum (diluted in PBST $1: 50 ; 1: 100$ or $1: 200)$ with the primary antibodies. Washing was done on drops of PBST three times for 10 min each. Afterwards the grids were incubated for $45 \mathrm{~min}$ on the secondary antibody (goat-anti-guinea pig IgG, coupled to $\sim 6 \mathrm{~nm}$ gold, Aurion), diluted 1:40 in PBST, and washed further as described above. Grids were stained with $3 \%$ (w/v) neutralized phosphotungstic acid for $1 \mathrm{~min}$, rinsed on drops of distilled water and blotted dry on filter paper.

\section{RESULTS}

\section{Immunolocalization of KcsA in the heterologeous $E$. coli host carrying a functional $\operatorname{kcs} A$ gene}

An E. coli host carrying the $k c s A$ gene with six histidine codons produces the resulting KcsA-Histag protein, which had previously been found to be predominantly located within the cytoplasmic membrane, from which it can be extracted using detergents (Schrempf et al., 1995; Meuser et al., 1999). The use of the intact KcsA protein (containing its two transmembrane helices) for generating antibodies resulted only in sera with relatively low titres. Therefore, as a prerequisite for immunological studies (for details see Methods), a stretch of the $k c s A$ gene corresponding to the Cterminus (60 amino acids) of the KcsA protein was cloned into an E. coli pQE32 vector. After optimization of the expression and isolation conditions, the corresponding protein was purified and used to raise antibodies (named anti-C-KcsA).

E. coli carrying the full-length $k c s A$ gene under control of the promoter of the lac operon (Schrempf et al., 1995), the truncated $k c s A$ gene (see above) or the E. coli control strain carrying the $\mathrm{pQE} 32$ vector were grown under inducing (IPTG) conditions. In extracts of the first strain proteins were detected (Fig. 2b, lane 3) corresponding to the 
(a)

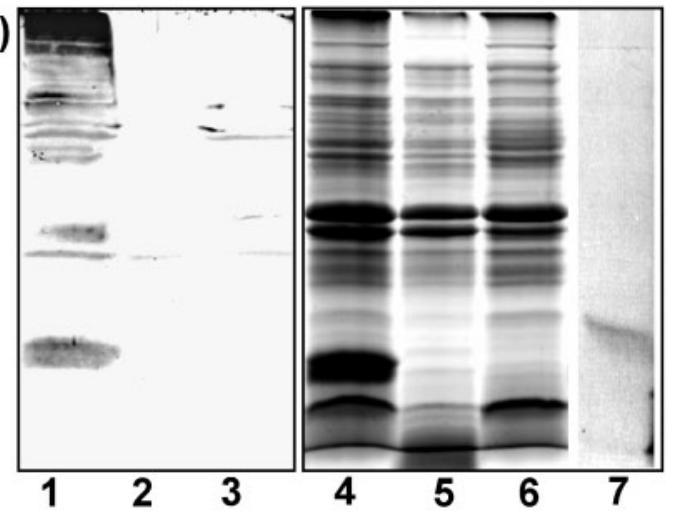

(b)

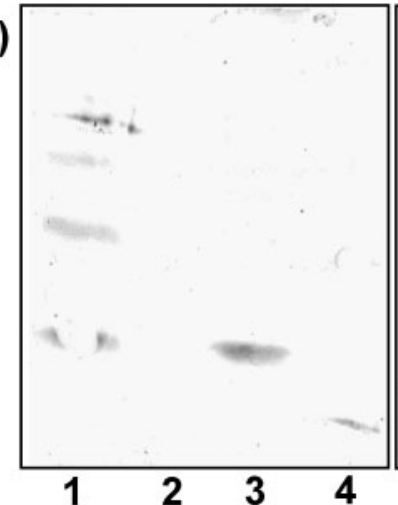

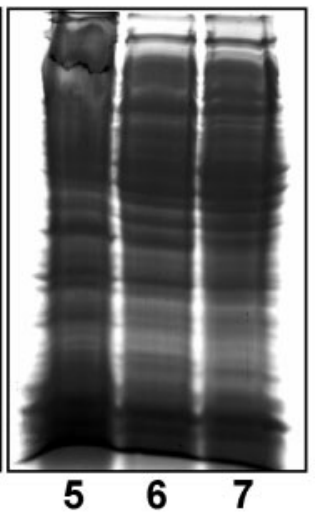

(c)

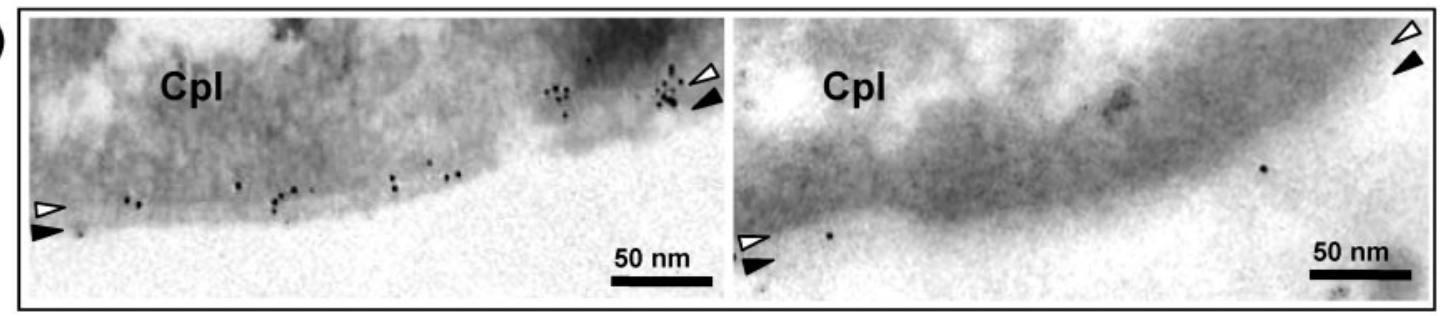

Vector $+k c s A$

Vector control

Fig. 2. Immunodetection of $\mathrm{KcsA}$ in $E$. coli. Cells of $E$. coli containing a pQE construct with the full-length (a, lanes 1,$4 ; b$, lanes 1,5$) k c s A$ gene, or with the truncated gene or the vector alone (a, lanes 2,5 ; b, lanes 2,6$)$ were harvested after induction with IPTG. After disruption of the cells, the membrane (a) and cytoplasmic (b) fractions were obtained. After separation by SDS-PAGE, gels were stained with Coomassie (a, lanes 4-7; b, lanes 5-7) or transferred to a nylon membrane and incubated with antibodies raised against the $\mathrm{C}$-terminus of $\operatorname{KcsA}(a$, lanes $1-3 ; b$, lanes 1-4). The purified full-length $\mathrm{Kcs}_{\mathrm{Histag}}$ protein served as control; note the oligomeric forms (a, lane $7 ; \mathrm{b}$, lane 1 ). (c) Induced E. coli cells carrying a pQE construct with the inserted $k c s A$ gene or having only the vector as control were embedded in Lowicryl K4M, ultrathin sectioned and afterwards treated with primary anti-KcsA antibodies and subsequently secondary (anti-guinea pig) goldlabelled antibodies. The outer face of the cell wall (black arrowheads), the cytoplasmic membrane (white arrowheads) and the cytoplasmic region (Cpl) are marked.

full-length KcsA ( $16 \mathrm{kDa})$. In cytoplasmic extracts from the E. coli strain carrying a construct encoding only the Cterminus of $\mathrm{KcsA}$, a protein band corresponding to the Cterminal portion $(6 \cdot 3 \mathrm{kDa})$ reacted with the antibodies (Fig. 2b, lane 4). Within extracts of the E. coli strain having only the vector, no reaction was detectable (Fig. 2b, lane 2). Within the membrane-containing fraction, the dominant portion of the full-length KcsA protein was present as monomers, dimers and oligomers (Fig. 2a, lanes 1 and 4). Due to lack of the transmembrane regions, the truncated KcsA protein (see above) was absent in the membrane fraction obtained (Fig. 2a, lane 3). The data revealed that the generated antibodies were specific for the designed KcsA protein.

For in situ studies, the above-described control strain carrying only the vector and the strain containing the fulllength $k c s A$ gene were grown as described above and embedded in resin. Ultrathin sections (see Methods) were treated with the antibodies raised against the C-terminal region of KcsA (anti-C-KcsA) and with secondary goldlabelled ( $\sim 6 \mathrm{~nm}$ ) antibodies. Inspection by electron microscopy revealed that the induced E. coli cells carrying the plasmid with the full-length $k c s A$ gene reacted with the antibodies. Gold particles bound near to the cytoplasmic membrane and close to the cytoplasm in smaller or larger clusters. Within sections of the control strain, the corresponding labelling was missing (Fig. 2c). The data clearly indicated that the antibodies detected KcsA within the cells.

\section{Immunolocalization reveals a clustered arrangement of KcsA in the cytoplasmic membrane of S. lividans hyphae}

As described previously (Schrempf et al., 1995), the kcsA gene was initially found within the chromosomal DNA of $S$. lividans wild-type (WT). To obtain a designed S. lividans mutant carrying an inactivated $k c s A$ gene (named $\Delta \mathrm{K}$ ), the WT strain was transformed with a pGM160-based construct which carried the Omega-hyg fragment (Blondelet-Rouault et al., 1997) including the hygromycin-resistance gene (hyg) with a translational terminator flanked by a few base pairs of the $5^{\prime}$ and $3^{\prime}$ ends, respectively, of the S. lividans kcsA gene connected to its upstream (698 bp) and downstream (639 bp) regions (see Methods and Fig. 1). Chromosomal DNA from several candidates was inspected for double 
(a)

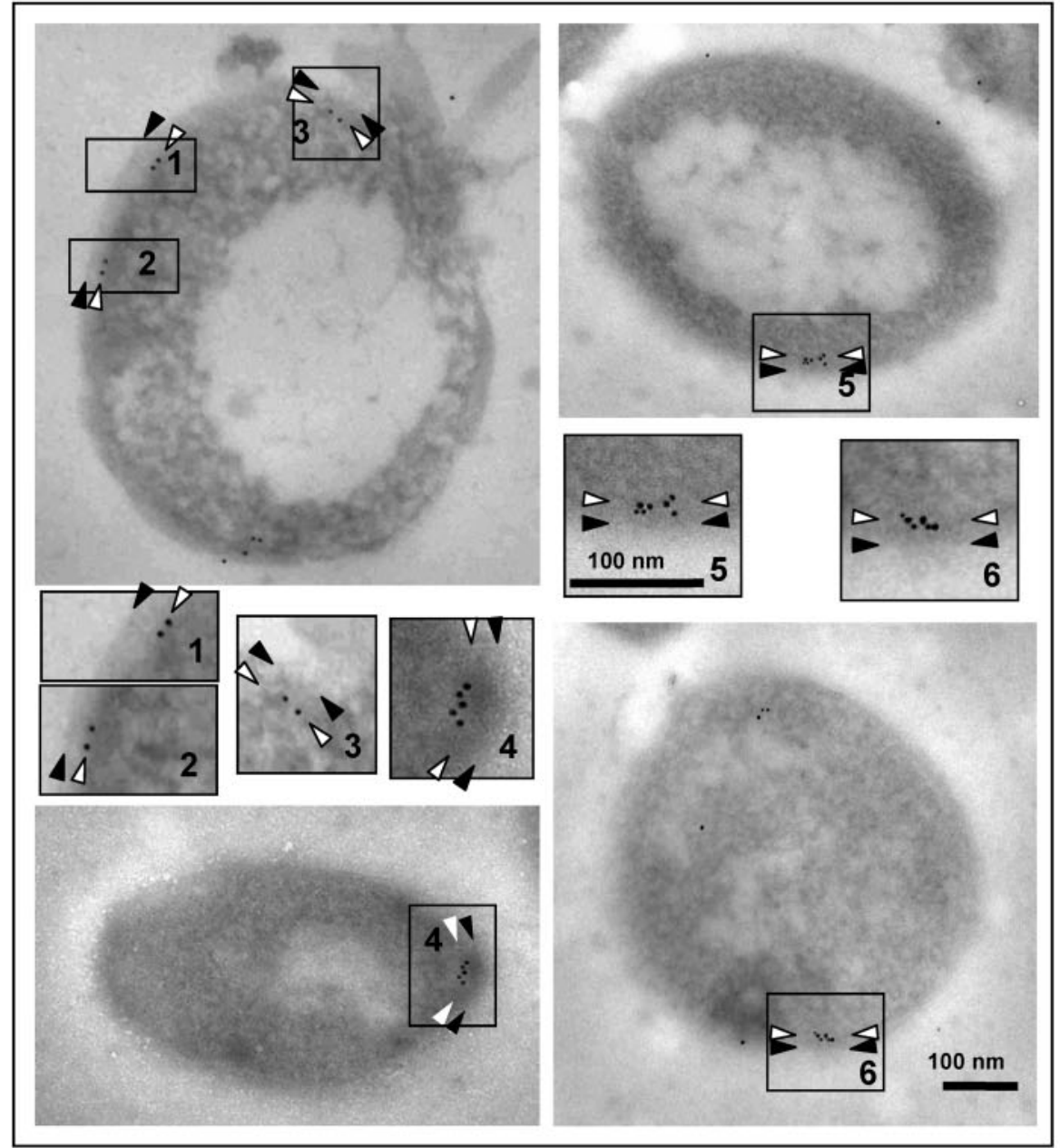

(b)

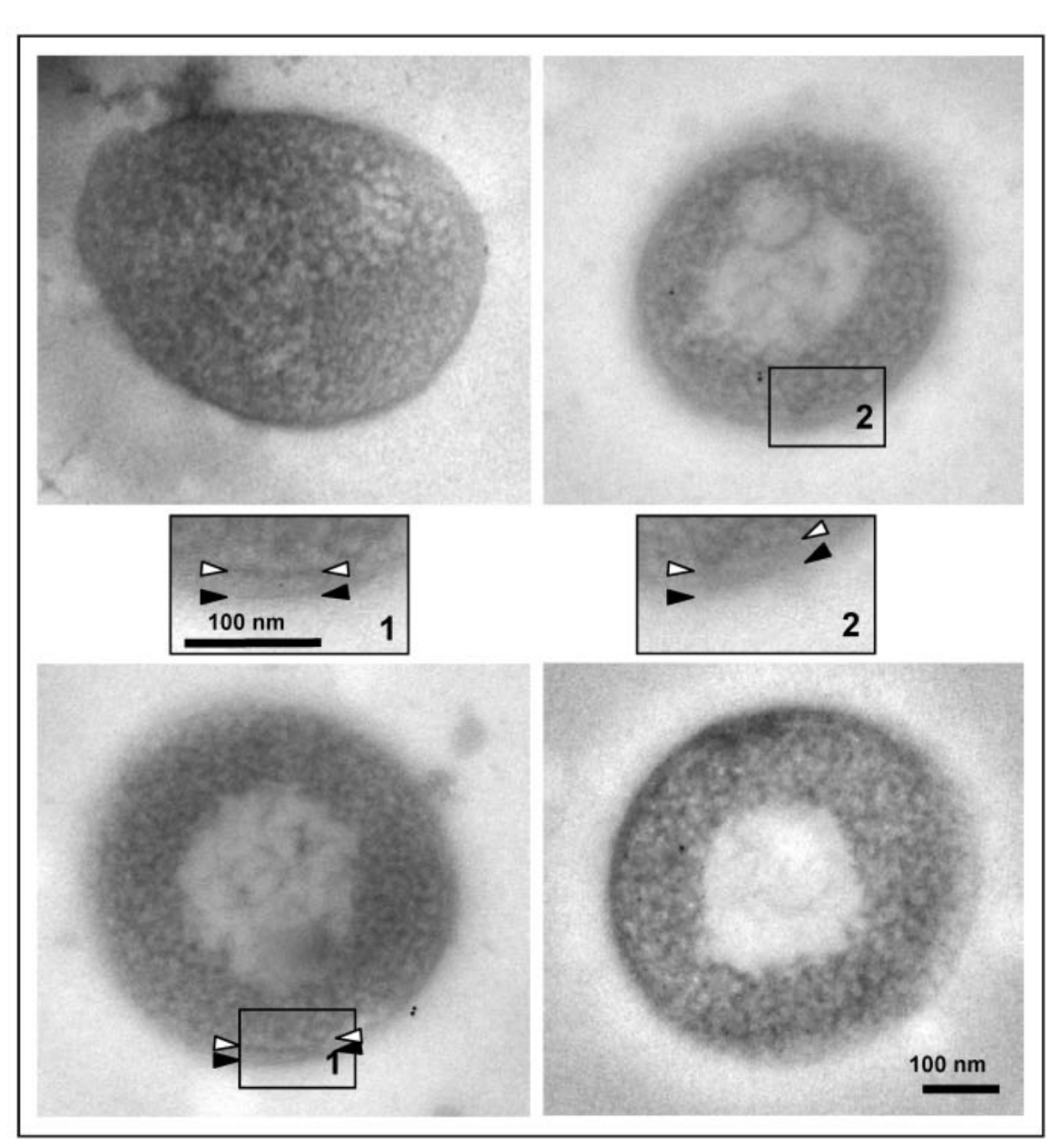

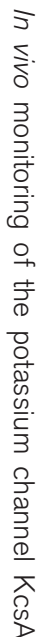

Fig. 3. Immunolocalization of KcsA in S. lividans. (a) WT strain; (b) $\Delta \mathrm{K}$ strain. S. lividans substrate hyphae were embedded in Lowicryl K4M. Ultrathin sections were incubated with primary anti-KcsA antibodies and subsequently secondary (anti-guinea pig) gold-labelled antibodies. Clusters of gold particles at the inner face of the cell wall, where the cytoplasmic membrane is located, are visible in the WT but absent in the $\Delta \mathrm{K}$ strain. The inner and outer face of the cell envelope are highlighted by white and black arrowheads, respectively. Several areas (numbered squares) have been enlarged to highlight the gold clusters. 
(a)

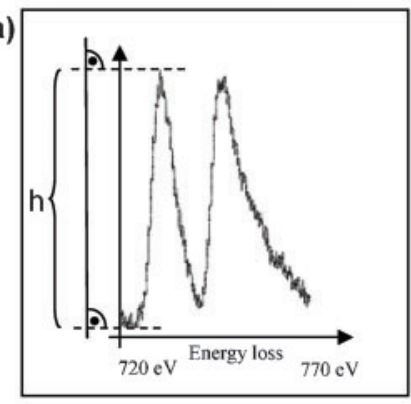

(b)

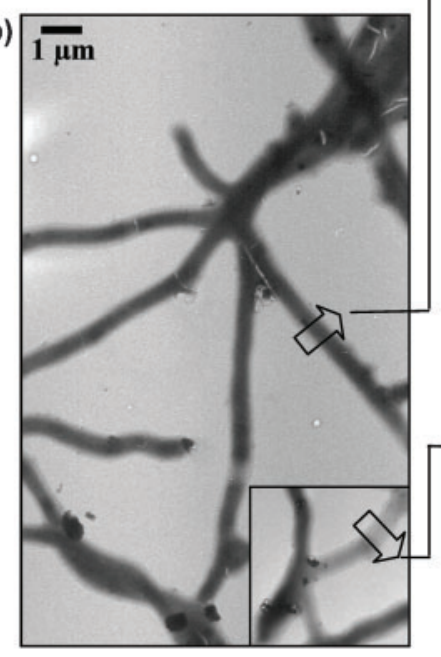

(c)

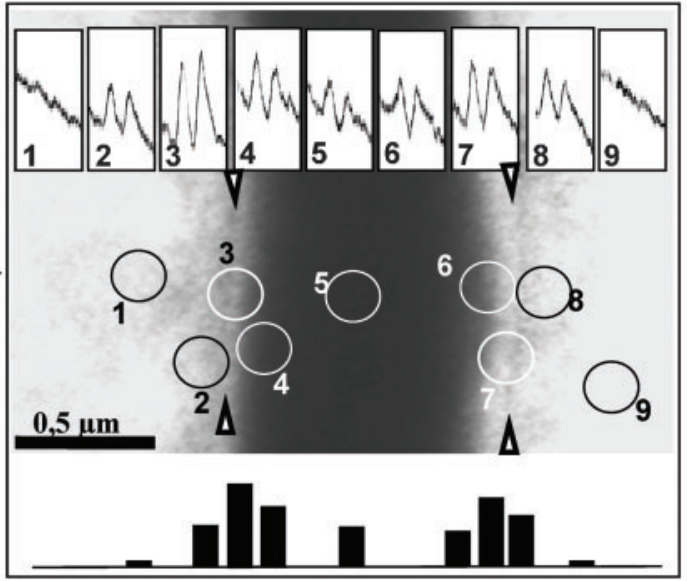

(d)

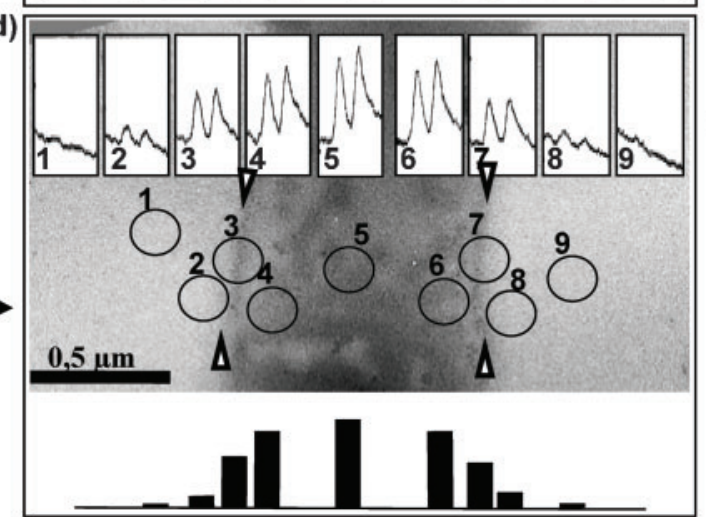

(e)

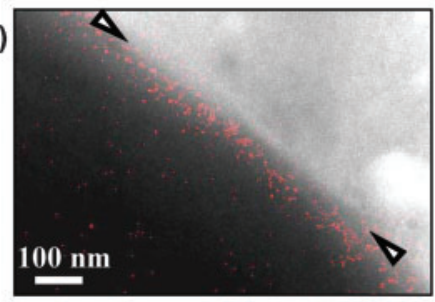

(f)

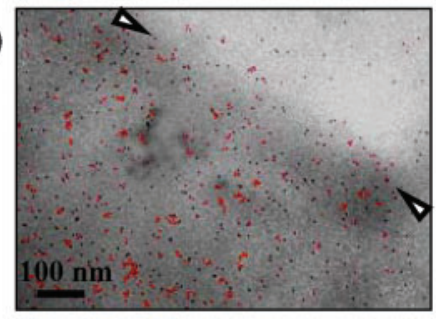

Fig. 4. Monitoring of caesium by EFTEM. (a) Reference EELS obtained from a CsCl crystal. A drop of saturated aqueous $\mathrm{CsCl}$ solution was air-dried on a Formvar-nickel grid, measuring was done on a crystal of $\sim 1 \mu \mathrm{m}$ size. (b) Hyphae of $S$. lividans showing intact hyphae (dark) and some hollow regions (bright, inset) derived from leakage of the cytoplasm. The proportion of leaked hyphae to intact ones is much smaller in the whole preparations than represented in this image. (c, d) Magnified view of parts of intact (c) and leaked (d) hyphae with the distribution of measuring points for EELS (circles) across them and illustration of the corresponding caesium-specific signals as spectra (above) and as a bar diagram according to the peak heights (below). The margins of the hyphae are marked between arrowheads. (e, f) ESI of the caesium distribution (red) around the margins (framed by arrowheads) of intact (e) or leaked (f) hyphae. The brighter half of the images corresponds to the adjacent support film.

crossover by using the hyg and the $k c s A$ gene as probes. Several hygromycin-resistant recombinants were found to contain the hyg gene (including a terminator) correctly inserted into the $k c s A$ gene (Fig. 1). One of them, named $\Delta \mathrm{K}$, was used here as negative control in the following immunolocalization experiments.

No labelling was found when whole hyphae of the S. lividans WT were treated with the above-described antibodies on grids or in a test tube (data not shown), as epitopes reacting with antibodies are hidden beneath the cell wall in vivo. Specific cross-reactivity is found only at the cytoplasmic membrane in sections of hyphae. Therefore a direct overall view on the distrubition of KcsA around and along the hyphae cannot be obtained using the immunogold technique. Within sections of the S. lividans WT hyphae, epitopes cross-reacting with the antibodies were found to be clustered within the cytoplasmic membrane. No such labelling was found in the $\Delta \mathrm{K}$ mutant (Fig. 3). The data clearly demonstrate that KcsA is localized in the S. lividans WT within the cytoplasmic membrane within separately spaced clusters. Using this technique, the exact spacing of clusters is difficult to estimate precisely, as not every epitope is expected to be labelled: fixation, dehydration and embedding in the resin probably decrease antigenicity and some epitopes are hidden inside the resin, inaccessible for antibodies. Nevertheless, the data allow the conclusion that in vivo the clusters can be at least $50 \mathrm{~nm}$ wide, probably wider. The mean distance between the clusters was estimated to be $500 \mathrm{~nm}$, as they were found in about $10 \%$ of the cross-sections of $50 \mathrm{~nm}$ thickness.

\section{EFTEM as a tool to analyse caesium distribution in hyphae of S. lividans WT}

Caesium can be recognized by EELS and the corresponding spectrum is characterized by the $\mathrm{M}_{45}$ edges at $726 \mathrm{eV}$ and 
(a)

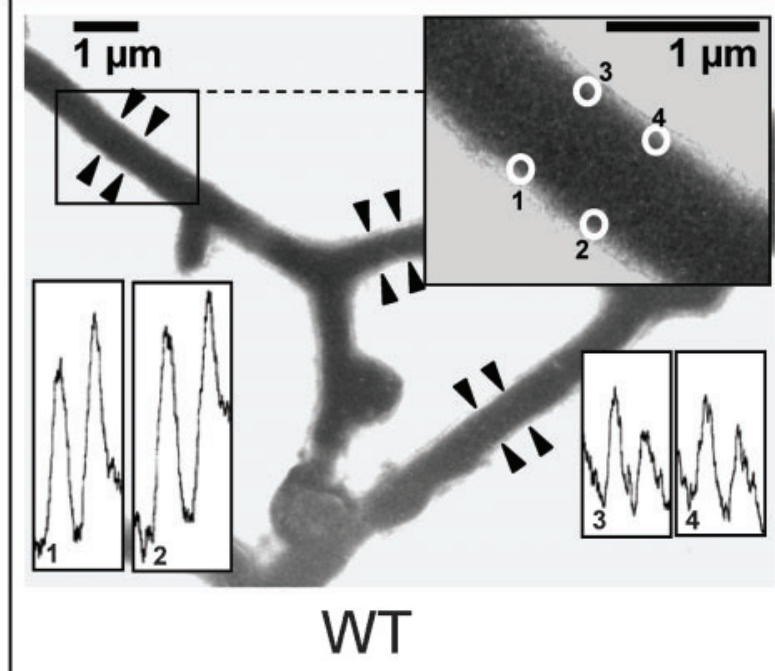

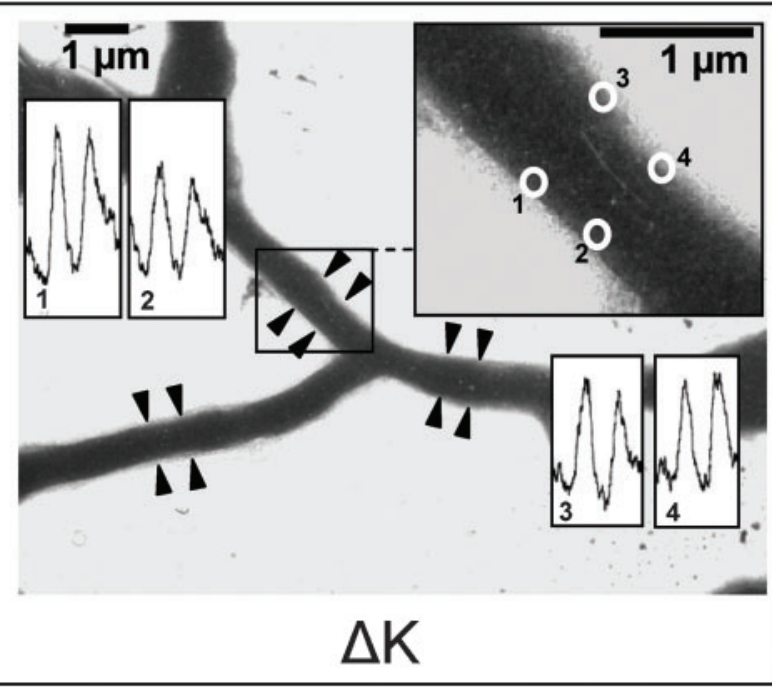

(b)

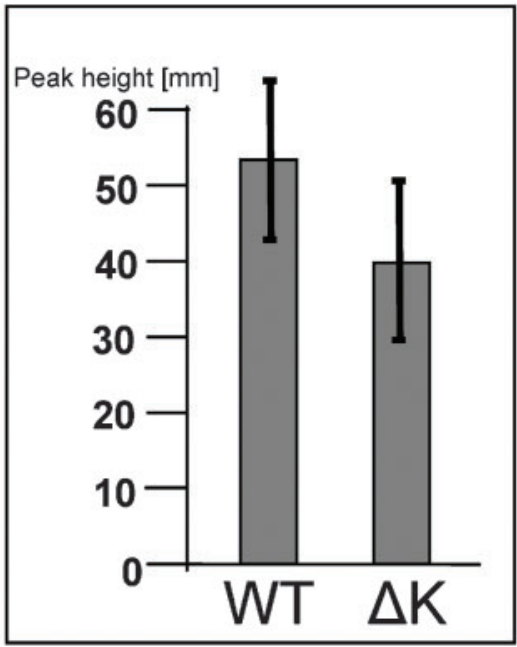

(c)

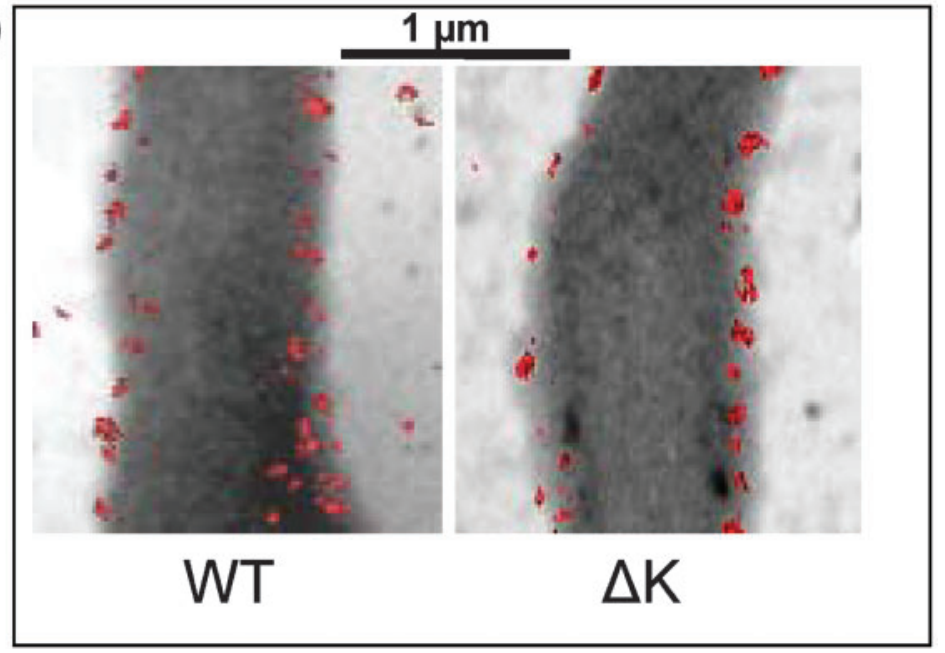

Fig. 5. Monitoring of caesium in S. lividans strains and evaluation procedure. (a) Analysis was done at various positions (arrowheads) along the hyphae using small measuring points arranged in groups of four, which are depicted as circles within the higher magnified insets. (b) For both the wild-type (WT) and $\Delta \mathrm{K}$ mutant, relative intensity (height in mm) of caesiumspecific peaks of 100 individual spectra was measured (as depicted in Fig. 4a). For both strains, the deduced mean value of the determined relative intensities for all measuring points is shown as a bar diagram with standard deviation. (c) Elemental map of caesium obtained by ESI analysis, using the same grids as for EELS. Caesium deposition (red) is visible at the margins of the hyphae in the WT and $\Delta \mathrm{K}$ mutant.

$740 \mathrm{eV}$ (Ahn \& Krivanek, 1983). Spectra achieved from CsCl crystals on reference grids (see Methods) showed these two characteristic peaks (Fig. 4a). The relative intensity (peak height) correlated with the amount of crystal material included in the measuring point (not shown). These peaks were absent in the control, a bare Formvar-nickel grid without $\mathrm{CsCl}$. This experiment showed that the method was suitable to differentiate among varying amounts of caesium being present in the measuring point. Therefore it was subsequently optimized for Streptomyces hyphae.

During preparation for transmission electron microscopy, the S. lividans hyphae mostly stay associated, forming a network on the support film. Intact hyphae appear dark. Due to air-drying, all hyphae collapse to a certain degree on the support film and the cytoplasm accumulates towards central regions, retracting from tips and margins. The latter appear comparatively bright as their optical density is lowered due to reduced levels of cytoplasm. Only a few hyphae get disrupted during sample preparation; these appear bright due to leakage of the cytoplasm.

S. lividans was grown under conditions which had been found to allow KcsA synthesis, mounted on grids and exposed to caesium ions (see Methods). After washing, the remaining caesium was monitored by EELS. Measuring 
points of 0.7 or $0.16 \mu \mathrm{m}$ diameter were positioned on the hyphae and, for controlling the background signal, also on the support film next to them. The background was found to be negligible. The large measuring points $(0 \cdot 7 \mu \mathrm{m}) \mathrm{did}$ not always include the exact diameter of the hyphae, as the latter varied between 0.5 and $0.8 \mu \mathrm{m}$. To ensure a constant portion of cellular material for every measurement, small measuring points of $0 \cdot 16 \mu \mathrm{m}$ were chosen.

Visualization of caesium by ESI revealed higher concentrations of caesium at the margins of hyphae compared to their central regions. This was confirmed by EELS using measuring points distributed in a row across the hyphae: signal intensities were always decreased in the central region, compared to the margins (Fig. 4b, c). In regions where the cytoplasm had leaked out from the hyphae (due to disruption during preparation) the cell envelope remained as a hollow tube, appearing bright due to complete loss of the cytoplasm. ESI revealed that in such areas the signal intensities were almost equal in all regions of the hyphae, as also observed by EELS (Fig. 4d). Artificial intrusion of caesium via disruptions can be ruled out as the signal intensities were not effectively higher in leaked than in intact hyphae. These observations can be explained as follows. During measuring, the electron beam passes the cell envelope, the cytoplasm, and once again the cell envelope. The recorded signal intensity correlates inversely with the amount of cytoplasm. The latter reduces the signal obtained from the cell envelope, due to its mass, and therefore obviously contains only low amounts of caesium in contrast to the cell envelope.

\section{S. lividans WT and $k \operatorname{cs} A$ mutant $\Delta K$ differ in the amount of caesium accumulation}

Using ESI and EELS, caesium was detected mostly at the margins of intact hyphae (Fig. 4). Visualization of caesium was possible by ESI; however, for comparable quantification the averaged peak height of many individual EEL spectra was found to be essential. The highest signals were obtained from small measuring points that were positioned centrally onto the margins, including one half of hypha material and one half of adjacent support film (Fig. 4c, measuring points $3+7$ ). In comparison, lower signal intensities were obtained by measuring exactly at the outside (including only adjacent support film, measuring points $2+8$ ) and also at the inside of the margins (including only hypha material, measuring points $4+6$ ). Probably a small fringe of caesium-containing medium surrounds the cells, due to capillary attraction. Based on these results, for all comparative studies the individual measuring points were always positioned exactly at the inner faces of the margins of the hyphae, and inclusion of adjacent support film was avoided. This procedure assured that only caesium directly associated with the cells contributed to the measurements, and any background signal was excluded.

To test whether the existence of KcsA in S. lividans hyphae has any effect on accumulation of caesium from the medium, we compared EELS data obtained from hyphae of the WT and the $\Delta \mathrm{K}$ mutant after exposure to caesium ions. Hyphae of the S. lividans WT and the corresponding $k c s A$ disruption mutant $\Delta \mathrm{K}$, lacking a functional $k c s A$ gene (Fig. 1), were transferred onto grids and exposed to $\mathrm{CsCl}$ as mentioned above (see Methods). Based on the results described in the previous paragraph, many small measuring points $(0 \cdot 16 \mu \mathrm{m})$ were placed at different intervals along the inner face of margins of the hyphae. As the signal intensity often differed to a certain degree between the two sides of the hyphae, pairs of measuring points were always positioned oppositely (Fig. 5a).

Individual spectra were evaluated for 100 measuring points $(0.16 \mu \mathrm{m})$ distributed along the hyphae of both S. lividans WT and mutant $\Delta \mathrm{K}$. Five different grids were used for each strain. The relative signal intensity of each individual spectrum was determined (see Methods). The deduced mean value for the WT was found to be $\approx 30 \%$ higher than for the $\Delta \mathrm{K}$ mutant (Fig. $5 \mathrm{~b}$ ).

\section{DISCUSSION}

As a prerequisite to localize KcsA channels within S. lividans hyphae, specific antibodies were generated. The C-terminal domain, which has so far no counterpart in other known proteins, has been found to have considerably higher immunogenicity than the full-length protein including its two membrane-spanning helices. Ultrathin sections of induced E. coli cells carrying the $k c s A$ gene in a multicopy vector (Schrempf et al., 1995) were treated with the anti-CKcsA antibodies followed by secondary gold-labelled antibodies. Rarely, unspecific gold labelling was detected on the outer face of the cell envelope from E. coli carrying the construct with the $k c s A$ gene; this feature was shared with the E. coli control cells containing only the vector plasmid (Fig. 2c). This control strain lacked, however, specific labelling at the cytoplasmic membrane. Gold labels were frequently found in clusters adjacent to each other within the cytoplasmic membrane and sometimes scattered within neighbouring regions in the cytoplasm (Fig. 2c). These findings are in agreement with previous results (Splitt et al., 2000) and the biochemical studies presented here (Fig. 2a, b), based on which it was shown that within the heterologous host the dominant portion of the full-length KcsA molecules is also extractable as monomers and oligomers from the E. coli membrane. Further studies had indicated that the monomers are directly interacting with the inner membrane in which the assembly to oligomeric forms was found to be independent of ATP hydrolysis. The protonmotive force was shown to be essential for the efficiency of oligomerization (van Dalen et al., 2000). Mutational analysis led to the identification of amino acid residues stablizing the tetrameric assembly (Splitt et al., 2000; Meuser et al., 2001).

Our comparative results (Fig. 2) unambiguously revealed that the generated antibodies were highly specific and 
suitable to detect KcsA in ultrathin sections of bacterial cells. Subsequently, longitudinal and cross-sections of hyphae of the S. lividans WT strain (containing a functional $k c s A$ gene) were treated with antibodies as outlined for the E. coli cells. Separate clusters of immunogold conjugates were localized close to the outer surface of the cytoplasm and the inner face of the cell wall (Fig. 3a). This region outlines the cytoplasmic membrane, which is in Gram-positive bacteria in close contact with the inner face of the cell wall. Due to the dimensions $(\sim 6 \mathrm{~nm})$ of the gold particles, it appears unlikely that each of the four C-termini within the KcsA tetramer, predicted to comprise in vivo one channel, will be labelled by a secondary gold-labelled antibody. Thus the identified clusters, usually containing three to seven gold particles, are very likely indicative of areas comprising up to several KcsA channels. Sections of the designed S. lividans $\Delta \mathrm{K}$ mutant, which lacks a functional $k c s A$ gene, do not exhibit specific cross-reactivity with the antibodies (Fig. 3b). Taken together, the data demonstrate what we believe to be the first in vivo localization of a bacterial $\mathrm{K}^{+}$ channel protein and verify that KcsA is located in the cytoplasmic membrane. This highly ordered clustering contrasts with the deregulated crowding and very heterogeneous assemblies observed within liposomes to which KcsA had been added (Molina et al., 2006).

For IEM, antibodies can be applied to a specimen before or after embedding and sectioning (i.e. pre-embedding or postembedding technique). Pre-embedding is generally more sensitive, but is suitable only for surface-accessible antigens. The bacterial cell wall is not permeable for antibodies, which is why membrane-located KcsA could only be labelled by post-embedding. IEM (using anti-peptide antibodies) was also applied to the KCNQ4 $\mathrm{K}^{+}$channel within the basal membrane of the hair cells of the mouse cochlea (Kharkovets et al., 2000) and the $\mathrm{K}^{+}$channels KCNQ1 and ERG1 within heart cells of rats (Rasmussen et al., 2004). Permeabilized human glioma cells allowed antibodies to access a chloride channel by pre-embedding (Olsen et al., 2003). The number of studies to localize transport proteins within bacteria is relatively low. The documented studies include the detection of a transport system involved in polysaccharide export within Pseudomonas aeruginosa via immunolabelling of lipopolysaccharides (Rocchetta \& Lam, 1997), and the localization of a proposed peptide transporter in the lipopolysaccharide layer of Synechocystis (Bölter et al., 1998).

Inspection of biological samples by EFTEM allows imaging of spatial elemental information. As KcsA is a potassium channel, it would have been reasonable to monitor potassium ions via EFTEM. However, the potassiumspecific signal is superimposed with that of carbon and therefore hardly detectable in biological material. A major difficulty in applying EFTEM is the loss of ions during sample preparation, especially during dehydration and embedding for ultrathin sectioning. Tracing of elements is most easily achieved if ions are bound tightly and focused to distinct cell loci. Metals deposited in electron-dense granules can be analysed by EELS very clearly (Liu \& Kottke, 2003). A combination of immunolabelling and EELS allowed mapping of the distribution of endocrine polypeptide hormones and proteins in pancreatic cells (Goping et al., 2003). Recent studies revealed distinct elemental maps within mussels, visualized by ESI (Bleher \& Machado, 2004). In Drosophila, lead and cereum ions could be analysed by EELS after their precipitation with phosphate which derived from degraded ATP, due to the activity of ATPase-dependent potassium pumps (Bohrmann \& Heinrich, 1994).

Within an artificial vesicle system (generated by fusing $S$. lividans protoplasts with liposomes), the KcsA potassium channel activity was found to be blocked by caesium ions (Schrempf et al., 1995). In contrast, the selectivity within the in vitro reconstituted KscA varied according to the different test conditions applied. These data suggest that the conformation of the reconstituted channels differs from that of those within the liposome-protoplast vesicles. This result was not surprising, as the artificial composition of the bilayer varies from those within the protoplasts (generated from the Streptomyces hyphae). In addition, the bilayer also lacks other components present in the natural cytoplasmic membrane. Hence it was concluded that the giant protoplast system mimics the physiological conditions most closely (Schrempf et al., 1995; Meuser et al., 1999). Competition of $\mathrm{Cs}^{+}$with $\mathrm{K}^{+}$had also been documented for ion-uptake systems in plants (Zhu \& Smolders, 2000). Based on these data, the S. lividans WT hyphae were exposed briefly to medium in which $\mathrm{KCl}$ had been replaced by $\mathrm{CsCl}$. It was expected that $\mathrm{Cs}^{+}$could enter the vestibule at KcsA channels (or a portion of them) opened during the exposure to $\mathrm{Cs}^{+}$.

Our data reveal that in S. lividans substrate hyphae, caesium ions are restricted mostly to the cell envelope, the outer and inner face of which can not be discriminated by EFTEM. The mean value (deduced from 100 evaluated areas) for caesium-specific signal intensity was $\approx 30 \%$ higher for the S. lividans WT than for the $\Delta \mathrm{K}$ mutant, lacking a functional $k c s A$ gene. Hence the determined enhanced value has to be attributed to the presence of KcsA within the WT. To a certain extent, unspecific binding of caesium to the outside of hyphae can be expected for both strains, independent of the presence of KcsA. In addition to this, the accumulation via other as yet uncharacterized transport systems seems likely. These effects are expected to be superimposed in the measurements for both strains.

The observed difference in caesium accumulation may also be due to cation sequestration by additional structures, associated with KcsA. Polyhydroxybutyrate (PHB) and inorganic phosphate (polyP) are widely distributed among pro- and eukaryotic organisms (see review by Reusch, 2000). $\mathrm{PHB}$ is a linear polymer of 3-hydroxybutyrate and is an amphiphilic polyester that forms ion-conducting complexes with salts. PolyP is a linear polymer of phosphoryl units and has the capacity for ion exchange and the ability to 
discriminate among cations by charge (Reusch, 1999). It has recently been suggested (Zakharian \& Reusch, 2004) that KcsA may recruit PHB and polyP to form a conductive core that selects and transports $\mathrm{K}^{+}$to the inner face of the selective filter.

In summary, our data reveal for the first time that the combination of IEM investigation and EFTEM is highly valuable for in vivo monitoring of ion channels and related proteins in bacteria.

\section{ACKNOWLEDGEMENTS}

We are very grateful to Dr H. Lünsdorf and Dr M. Hoppert for excellent advice to use EFTEM and we thank D. Müller for support in cultivating strains. The work was partly financed from a grant to H. Schrempf given by Deutsche Forschungsgemeinschaft (SFB 431, P4).

\section{REFERENCES}

Ahn, C. C. \& Krivanek, O. L. (1983). A reference guide of electron energy loss spectra covering all stable elements. Warrendale, PA: Gatan.

Bleher, R. \& Machado, J. (2004). Paracellular pathways in the shell epithelium of Anodonta cygnea. J Exp Zool 301, 419-427.

Blondelet-Rouault, M. H., Weiser, J., Lebrihi, A., Branny, P. \& Pernodet, J. L. (1997). Antibiotic resistance gene cassettes derived from the omega interposon for use in E. coli and Streptomyces. Gene 190, 315-317.

Bohrmann, J. \& Heinrich, U. R. (1994). Localization of potassium pumps in Drosophila ovarian follicles. Zygote 2, 189-199.

Bölter, B., Soll, J., Schulz, A., Hinnah, S. \& Wagner, R. (1998). Origin of a chloroplast protein importer. Proc Natl Acad Sci U S A95, 15831-15836.

Bucking, H., Beckmann, S., Heyser, W. \& Kottke, I. (1998). Elemental contents in vacuolar granules of extomycorrhizal fungi measured by EELS and EDXS. A comparison of different methods and preparation techniques. Micron 29, 53-61.

Chavez, F., Lünsdorf, H. \& Jerez, C. A. (2004). Growth of polychlorinated-biphenyl-degrading bacteria in the presence of biphenyl and chlorobiphenyls generates oxidative stress and massive accumulation of inorganic polyphosphate. Appl Environ Microbiol 70, 3064-3072.

Cruz, A. F. (2004). Element storage in spores of Gigaspora margarita Becker \& Hall measured by electron energy loss spectroscopy (EELS). Acta Bot Bras 18, 473-480.

Derst, C. \& Karschin, A. (1998). Evolutionary link between prokaryotic and eukaryotic $\mathrm{K}^{+}$channels. J Exp Biol 201, 2791-2799.

Doyle, D. A. (2004). Structural themes in ion channels. Eur Biophys $J$ 33, 175-179.

Doyle, D. A., Cabral, J. M., Pfuetzner, R. A., Kuo, A., Gulbis, J. M., Cohen, S. L., Chait, B. T. \& MacKinnon, R. (1998). The structure of the potassium channel: molecular basis of $\mathrm{K}^{+}$conduction and selectivity. Science 280, 69-77.

Durell, S. R. \& Guy, H. R. (2001). A family of putative Kir potassium channels in prokaryotes. BMC Evol Biol 1, 14.

Durell, S. R., Hao, Y., Nakamura, T., Bakker, E. P. \& Guy, H. R. (1999). Evolutionary relationship between $\mathrm{K}^{+}$channels and symporters. Biophys J 77, 775-788.
Goping, G., Pollard, H. B., Srivastava, M. \& Leapman, R. (2003). Mapping protein expression in mouse pancreatic islets by immunolabeling and electron energy loss spectrum-imaging. Microsc Res Tech 61, 448-456.

Heinrich, U. R., Maurer, J. \& Mann, W. (1998). Possible $\mathrm{Ca}^{2+}$. dependent mechanism of apical outer hair cell modulation within the cochlea of the guinea pig. Cell Tissue Res 292, 57-65.

Hofer, F., Grogger, W., Kothleitner, G. \& Warbichler, P. (1999). Quantitative compositional imaging with energy-filtering TEM. In EMAG'99, 25-27 August 1999, Sheffield, UK (http://www.felmi-zfe. tugraz.at/download/EMAG99final.pdf).

Hopwood, D. A., Bibb, M. J., Chater, K. F. \& 7 other authors (1985). Genetic Manipulation of Streptomyces: a Laboratory Manual. Norwich: John Innes Foundation.

Kharkovets, T., Hardelin, J. P., Safieddine, S., Schweizer, M., El Amraoui, A., Petit, C. \& Jentsch, T. J. (2000). KCNQ4, a $\mathrm{K}^{+}$channel mutated in a form of dominant deafness, is expressed in the inner ear and the central auditory pathway. Proc Natl Acad Sci U S A 97, 4333-4338.

Kottke, I. (1991). Electron energy loss spectroscopy and imaging technique for subcellular localization of elements in mycorrhiza. Methods Microbiol 23, 369-382.

Kutzner, H. J. (1981). The family Streptomycetaceae. In The Prokaryotes: a Handbook on Habitats, Isolation and Identification of Bacteria, pp. 2028-2090. Edited by M. P. Starr, H. Stolp, H. G. Trüper, A. Balows \& H. Schlegel. Berlin: Springer.

Liu, D. \& Kottke, I. (2003). Subcellular localization of chromium and nickel in root cells of Allium cepa by EELS and ESI. Cell Biol Toxicol 19, 299-311.

Meuser, D., Splitt, H., Wagner, R. \& Schrempf, H. (1999). Exploring the open pore of the $\mathrm{K}^{+}$channel KcsA from Streptomyces lividans. FEBS Lett 462, 447-452.

Meuser, D., Splitt, H., Wagner, R. \& Schrempf, H. (2001). Mutations stabilizing an open conformation within the external region of the permeation pathway of the potassium channel KcsA. Eur Biophys $J$ 30, 385-391.

Molina, M. L., Barrera, F. N., Fernandez, A. M., Poveda, J. A., Renart, M. L., Encinar, J. A., Riquelme, G. \& Gonzalez-Ros, J. M. (2006). Clustering and coupled gating modulate the activity in KcsA, a potassium channel model. J Biol Chem (http://www.jbc.org/cgi/doi/ 10.1074/jbc.M600342200).

Muth, G., Farr, M., Hartmann, V. \& Wohlleben, W. (1995). Streptomyces ghanaensis plasmid pSG5: nucleotide sequence analysis of the self-transmissible minimal replicon and characterization of the replication mode. Plasmid 33, 113-126.

Olsen, M. L., Schade, S., Lyons, S. A., Amaral, M. D. \& Sontheimer, H. (2003). Expression of voltage-gated chloride channels in human glioma cells. J Neurosci 23, 5572-5582.

Rasmussen, H. B., Moller, M., Knaus, H. G., Jensen, B. S., Olesen, S. P. \& Jorgensen, N. K. (2004). Subcellular localization of the delayed rectifier $\mathrm{K}^{+}$channels KCNQ1 and ERG1 in the rat heart. Am J Physiol Heart Circ Physiol 286, H1300-H1309.

Reusch, R. N. (1999). Streptomyces lividans potassium channel contains poly- $(R)$-3-hydroxybutyrate and inorganic polyphosphate. Biochemistry 38, 15666-15672.

Reusch, R. N. (2000). Transmembrane ion transport by polyphosphate/poly-(R)-3-hydroxybutyrate complexes. Biochemistry (Mosc) 65, 280-295.

Rocchetta, H. L. \& Lam, J. S. (1997). Identification and functional characterization of an $\mathrm{ABC}$ transport system involved in polysaccharide export of A-band lipopolysaccharide in Pseudomonas aeruginosa. J Bacteriol 179, 4713-4724. 
Roth, J., Bendayan, M., Carlemalm, E., Villiger, W. \& Garavito, M. (1981). Enhancement of structural preservation and immunocytochemical staining in low temperature embedded pancreatic tissue. J Histochem Cytochem 29, 663-671.

Sambrook, J., Fritsch, E. F. \& Maniatis, T. (1989). Molecular Cloning: a Laboratory Manual, 2nd edn. Cold Spring Harbor, NY: Cold Spring Harbor Laboratory.

Schlochtermeier, A., Niemeyer, F. \& Schrempf, H. (1992). Biochemical and electron microscopic studies of the Streptomyces reticuli cellulase (Avicelase) in its mycelium-associated and extracellular forms. Appl Environ Microbiol 58, 3240-3248.

Schrempf, H. (1999). Investigations of streptomycetes using tools of recombinant DNA technology. In Manual of Industrial Microbiology and Biotechnology, 2nd edn, pp. 501-510. Edited by A. L. Demain \& J. E. Davies. Washington, DC: American Society for Microbiology.

Schrempf, H. (2005). Deciphering the Streptomyces lividans KcsA as a channel model. In Bacterial Ion Channels and Their Eukaryotic Homologs, pp. 14-67. Edited by A. Kubalski \& B. Martinac. Washington, DC: American Society for Microbiology.

Schrempf, H., Schmidt, O., Kümmerlen, R., Hinnah, S., Müller, D., Betzler, M., Steinkamp, T. \& Wagner, R. (1995). A prokaryotic potassium ion channel with two predicted transmembrane segments from Streptomyces lividans. EMBO J 14, 5170-5178.

Somlyo, A. P. \& Shuman, H. (1982). Electron probe and electron energy loss analysis in biology. Ultramicroscopy 8, 219-233.

Splitt, H., Meuser, D., Borovok, I., Betzler, M. \& Schrempf, H. (2000). Pore mutations affecting tetrameric assembly and functioning of the potassium channel KcsA from Streptomyces lividans. FEBS Lett 472, 83-87.

van Dalen, A., Schrempf, H., Killian, J. A. \& de Kruijff, B. (2000). Efficient membrane assembly of the KcsA potassium channel in Escherichia coli requires the protonmotive force. EMBO Rep 1, 340-346.

Xiao, X., Wang, F., Saito, A., Majka, J., Schlösser, A. \& Schrempf, H. (2002). The novel Streptomyces olivaceoviridis ABC transporter Ngc mediates uptake of $\mathrm{N}$-acetylglucosamine and $\mathrm{N}, \mathrm{N}^{\prime}$-diacetylchitobiose. Mol Genet Genomics 267, 429-439.

Zakharian, E. \& Reusch, R. N. (2004). Functional evidence for a supramolecular structure for the Streptomyces lividans potassium channel KcsA. Biochem Biophys Res Commun 322, 1059-1065.

Zhu, Y. G. \& Smolders, E. (2000). Plant uptake of radiocaesium: a review of mechanisms, regulation and application. J Exp Bot 51, 1635-1645. 